\title{
Investigation into clustering of synthetic jet actuators for flow separation control applications
}

\author{
S. C. Liddle and N. J. Wood \\ School of Mechanical, Aerospace and Civil Engineering \\ University of Manchester \\ Manchester, UK
}

\begin{abstract}
An investigation into the behaviour of clustered synthetic jet Actuators for flow-control applications is described. Experiments have been undertaken with two small-scale synthetic jet actuators in a zero-pressure gradient boundary-layer, in order to investigate the effect of configuration yaw angle and relative input signal phase. Oil-flow visualisation and hotwire anemometry techniques were used, demonstrating that changes in the downstream flow structure could be observed. Compared to a streamwise configuration, in which a symmetrical counter-rotating vortex pair was produced by the synthetic jet-boundary-layer interaction, a broader asymmetric interaction was produced in a $15^{\circ}$ yaw configuration. Streamwise velocity contour plots, illustrating the development of the interaction downstream, over four phase angles, were presented. Significant differences in the PSD analyses of downstream streamwise velocity time histories were found, suggesting that input signal phase could influence the stability and hence effectiveness of flow structures used in flow-control applications.
\end{abstract}

\section{NOMENCLATURE}

\footnotetext{
$d_{c} \quad$ cavity diameter

$d_{o} \quad$ orifice diameter

$d_{p z t} \quad$ diameter of piezo-ceramic element

$d_{\text {shim }}$ diameter of diaphragm

$h \quad$ cavity height

$R \quad$ velocity ratio, $U_{j e l} / U$
}

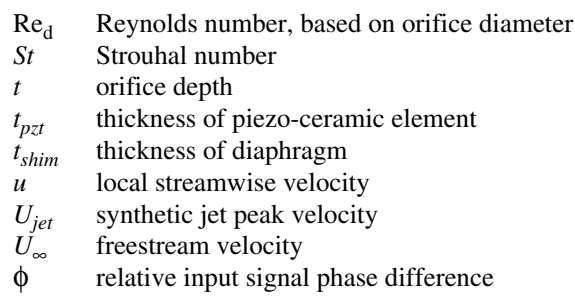

\subsection{INTRODUCTION}

The control of flow separation is of great importance in the aerodynamic design of flight vehicles. In recent years, emphasis has been placed on the use of active devices that can be used to manipulate the boundary layer and be tuned to the operating flow condition in order to maximise performance. In this way, advantages are offered 

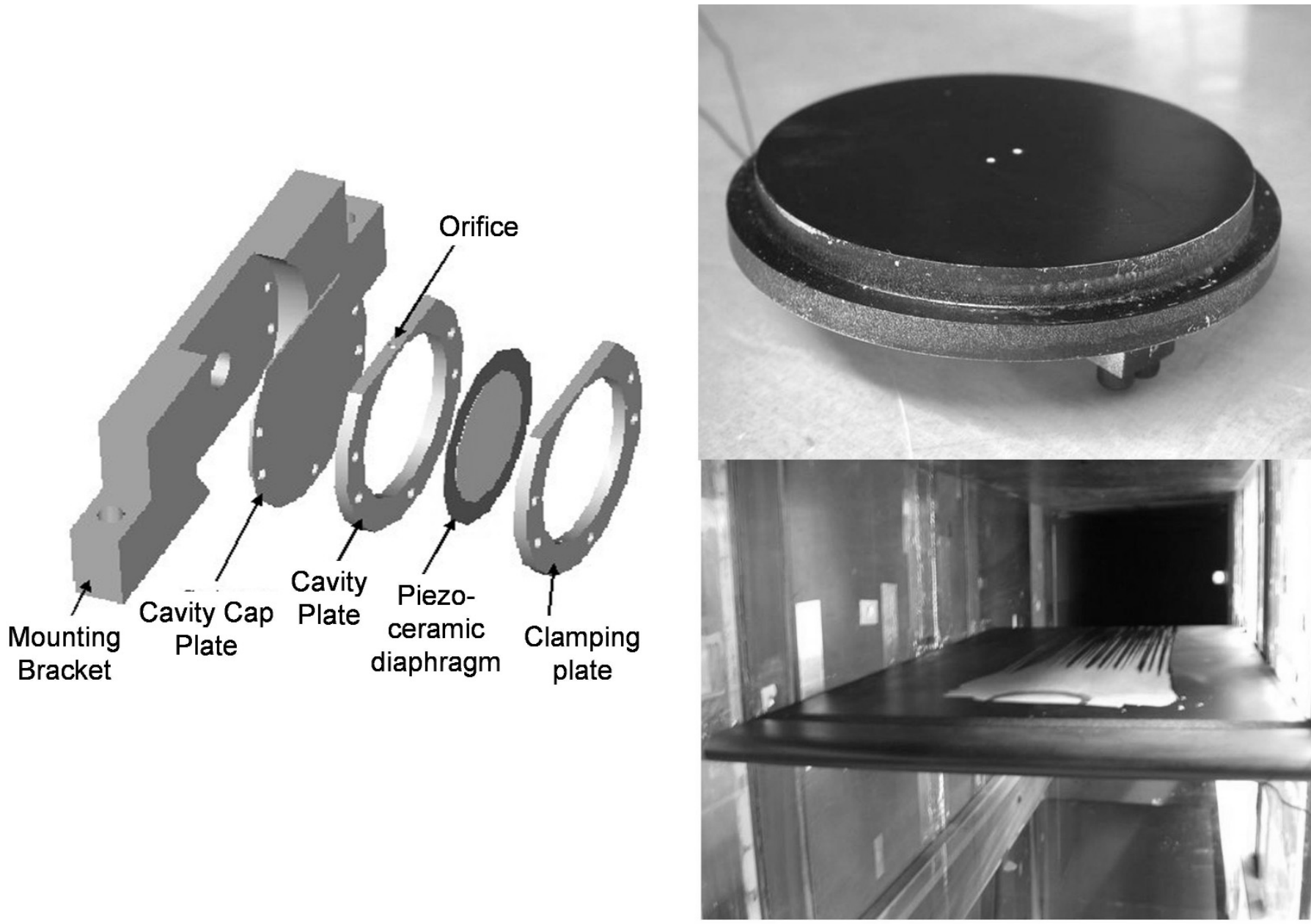

Figure 1. Illustration of small-scale synthetic actuator zero pressure gradient turbulent boundary layer rig. Clockwise from left: Exploded view of one actuator; two such actuators mounted in the circular plate; Circular plate assembly mounted in the flat-plate aerofoil, and in the $0.5 \mathrm{~m} \times 0.5 \mathrm{~m}$ Goldstein tunnel.

over traditional passive separation control methods, of which Vanetype vortex generators are an example.

The work described here forms part of a flow control project for civil transport aircraft aileronsz. Typically, these are deflected down in the take-off and landing configuration, in order to augment the high-lift system. The challenge of providing the required lateral control effectiveness, while the aileron are forced to operate around a downwards-deflected datum point, is an ideal application for active flow control. In this case, the requirement for use only during highlift operation, coupled with the sealed design of the aileron-wingbox interface, implies the use of technology that does not alter the external profile. Additionally, the outboard location implies that weight penalties would result from any requirement for engine bleed air transfer. These design drivers have led to the proposed use of synthetic jet actuators (SJAs).

To date, work has been reported from many sources on the use of SJA technology for aerofoil separation control applications (see for example Refs 1 and 2). This has included data on the augmentation of plain flap performance by the use of actuators with orifices mounted near to the hinge line, on the upper surface. In general, the results reported are for single, high aspect-ratio rectangular orifices. However, the use of discreet circular orifices has been demonstrated and may be considered to have some advantages in the production of coherent vortex rings ${ }^{(3)}$. Additionally, the potential exists to improve the penetration, and hence the downstream mixing potential, of the resulting vortex structures by effective clustering of discreet orifices.
Previous work has investigated the effect of orienting arrays of high-aspect ratio rectangular ${ }^{(4) 5}$ and circular $^{(6)}$ orifices. The current work aims to expand on these programmes of fundamental research by investigating the effect of altering input signal phase for closely clustered actuators.

\subsection{EXPERIMENTAL APPROACH}

The investigation was conducted in the Goldstein Tunnel at the Manchester School of Engineering's Goldstein Research Laboratory. This facility is a closed-return wind tunnel with a $0.5 \mathrm{~m} \times 0.5 \mathrm{~m}$ working section, and turbulence levels lower than $0.1 \%$. The tunnel is equipped with a Dantec traverse mechanism, designed for hotwire measurements. Both to allow comparison with previous experiments and to remain well within the operating range of the tunnel, a freestream velocity, $U_{\infty}=28 \mathrm{~ms}^{-1}$ was used throughout. This gave a Reynolds number based on orifice diameter, $\operatorname{Re}_{\mathrm{d}}=2,261$ and Strouhal number $S t=0.069$.

The Flat-plate aerofoil model spans the width of the tunnel, being mounted at approximately mid-height in the downstream working section. The model is equipped with an elliptical cross-section leading edge, providing an attached boundary layer. Immediately behind this is a grit strip, ensuring and fixing transition. The SJA orifices are approximately $110 \mathrm{~mm}$ downstream of the grit strip (variable with yaw angle). A zero-pressure gradient is established by 


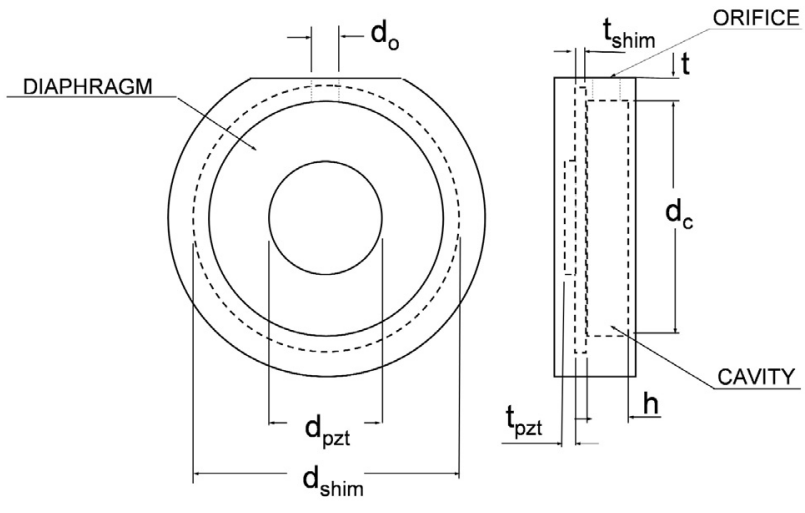

Figure 2. Schematic of a synthetic jet actuator, defining the salient dimensions as referred to in Table 1.

running the tunnel at maximum speed, and altering the setting of the flexible roof of the working section. The roof features an array of 18 static tappings, eight of which occur over the model. These pressures are indicated on a multi-tube manometer.

The use of a zero-pressure gradient boundary layer in this study was driven by a desire to compare the data gained with previous work $^{(6,7)}$ conducted using similar techniques. Furthermore, the aim of the work was to investigate the interactions between synthetic jet flows and a turbulent boundary layer, before introducing a pressure gradient as an additional parameter. A likely configuration for aileron separation control would see an array of actuators mounted behind the rear spar of the outboard wing on a transport aircraft, with the chordwise position of the orifices on the upper wing surface in the range $65 \%-80 \%$. The relatively flat-topped, supercritical aerofoils typically used in such applications have low adverse pressure gradients in this region at typical take-off and landing incidences (see, for example Ref. 8); it was thought therefore that the objective of characterising the interactions between close-coupled actuators in a zero pressure gradient was valuable when the potential application is considered.

The SJAs themselves are manufactured in-house, with the exception of the piezo-ceramic diaphragms, which are sourced commercially. Two actuator assemblies are mounted into a circular plate, with interchangeable plates allowing variable orifice spacing. In this case, the spacing has been fixed at $7 \mathrm{~mm}$. This complete assembly is then located in a recess in the flat plate model, attached by means of bespoke clamps and butterfly nuts. An exploded view of the actuator assembly, together with an illustration of the complete model mounted in the Goldstein Tunnel, is given in Fig. 1. Dimensions are given in Table 1, referring to the generic piezoceramic diaphragm-driven SJA illustrated in Fig. 2.

The input signal is supplied to the actuator via a small cable soldered to the piezo-ceramic disk. The return path is through the conductive brackets, with a cable simply wrapped around the bolt. The input signal itself is a sine wave, as previous experience with a square wave signal was considered to fatigue the diaphragms too quickly. The coordinate system used and referred to subsequently has its origin at the mid-point between the two orifices, as illustrated in Fig. 3.

The requirement for a versatile and efficient phase-shifting system has driven the use of a PC-controlled digital-to-analogue card (DAC) for control of the SJAs, using National Instruments LabView software for the development of virtual instrument control. Both CTA data acquisition and SJA power and control systems used a single measurement computing PCI-DAS6402 16-bit card, which featured both ADC and DAC capabilities.

The DAC output signals are fed to individual power amplifiers, with $\pm 55 \mathrm{v}$ capabilities. Both outputs are monitored using an

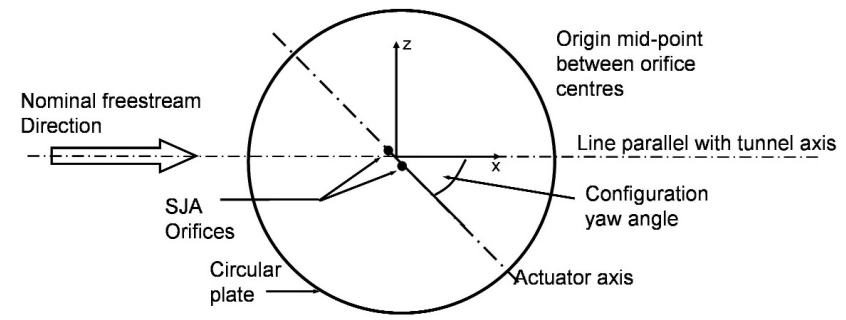

Figure 3: Schematic of circular plate, defining coordinate system.

oscilloscope and digital voltmeters, giving indications of frequency and phase.

The peak output of the SJAs, in terms of maximum velocity achieved, occurred at the system resonant frequency of $\sim 1,600 \mathrm{~Hz}$. From the method quoted in Ref. 9, the Helmholtz frequency of the cavity was $1,842 \mathrm{~Hz}$. In the initial flow-visualisation series of experiments, the actuation frequency was chosen to give matched peak velocities between the two actuators; this required a frequency of $1,950 \mathrm{~Hz}$ to be used. This was necessary as a single driving signal was used for both actuators, and a facility to allow variation in voltage to account for differing actuator characteristics was unavailable. In the case of the CTA experiments, the availability of PC-based control allowed velocity matching at a fixed frequency, and they were therefore operated at $1,600 \mathrm{~Hz}$. The role played by actuation frequency has been shown to be critical in separation control applications ${ }^{(10)}$, where dimensionless frequency is associated with the length scales of the separated region. However, in this study the limited change between these synthetic jet frequencies, in the attached boundary layer, has little effect.

The baseline configuration is that with the array axis parallel to the tunnel span. Description here is given of work involving two alternative configurations: Configuration 2 , in which the array axis is at $15^{\circ}$ to the streamwise axis, and Configuration 3 , in which the streamwise and array axes are coincident.

The resulting flow fields were investigated using both oil-flow visualisation and CTA anemometry. In the former case, a 6:1 kerosene/fluorescent dye mixture was used to detect the surface flows downstream of the actuators. Images of the resulting flow patterns were recorded using a digital camera mounted on a small tripod; illumination was provided by an ultra-violet tube mounted in a standard strip light fitting. This enabled the relative effect of the different configurations on the development of boundary layer structures to be assessed, before a region for mapping by hotwire was established.

Table 1

Synthetic jet actuator dimensions
Dimension

$d_{o}$

$t$

$h$

$d_{c}$

$d_{p z t}$

$d_{\text {shim }}$

$t_{p z t}$

$t_{\text {shim }}$
Value (mm)

$1 \cdot 2$

$0 \cdot 7$

$2 \cdot 0$

25.4

$20 \cdot 0$

$27 \cdot 0$

$0 \cdot 1$

$0 \cdot 1$ 


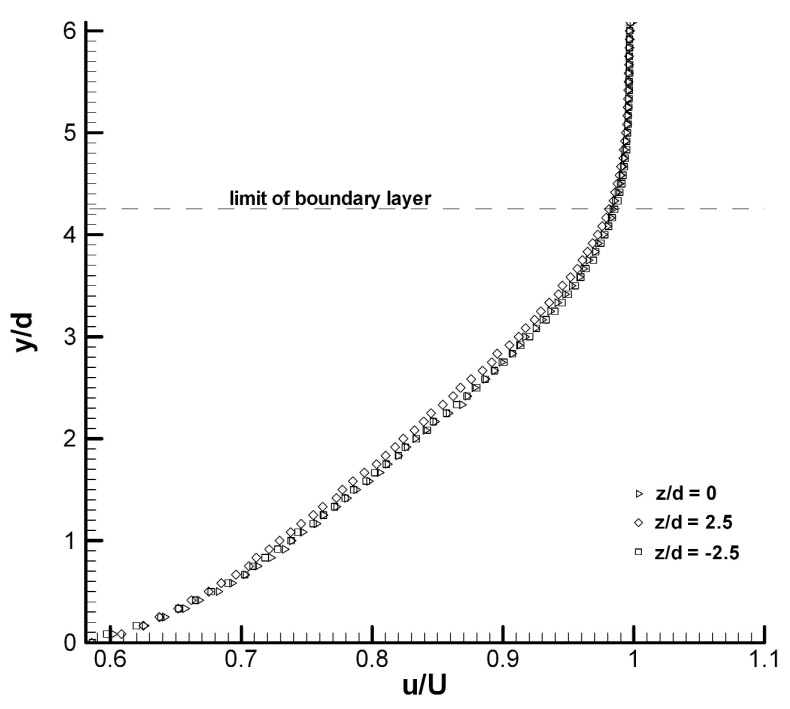

Figure 4. Boundary layer mean velocity profile, measured at $x / d_{0}=10$ at three spanwise locations. $U_{\infty}=28 \mathrm{~ms}^{-1}$.

The CTA experiments were conducted using a Dantec SN hotwire anemometer probe, mounted in the previously mentioned traverse mechanism, and connected to Dantec bridge amplifier and signal conditioning units. In this case, the hotwire probe was of the boundary layer type (Dantec 55P15), and mounted on a curved holder in order to allow investigation close to the wall. As the objective was to investigate the developed downstream flow and given the flow patterns found in the oil-flow visualisations, the measurement space was fixed (non-dimensionally) as $10 d_{0} \leq \times \leq 25 d_{\mathrm{o}}$, at increments of $5 d_{\mathrm{o}}$. Four measurement $y z$-planes were therefore created. The $y$-axis was traversed in $0.1 \mathrm{~mm}$ increments, using a motorised drive under software control. Due to a motor failure on the z-axis drive, manual movement was necessary, practically limiting resolution to $1 \mathrm{~mm}$, in the range $-4 \cdot 167 d_{0} \leq z \leq 4 \cdot 167 d_{0}$. Each measurement plane consisted of 814 data points. The CTA data were sampled using the previously mentioned Measurement Computing data acquisition card; 10,000 samples at a rate of $10 \mathrm{kHz}$ were collected for each data point.

In order to confirm the accuracy of the oil-flow visualisation results, a minimum of four images were compared for each case considered. The technique has some limitations: it can show only the time-averaged motion and the results will incorporate any unsteady effects of the experimental start-up procedure. However, the results gained can be considered accurate enough to adequately display salient features of the near-wall flow, and hence provide an indication for the direction of future investigation.

The accuracy of the CTA measurement is affected by the use of a single wire, as this technique cannot resolve the components of the three-dimensional flow and therefore provides an indication only of total velocity. However, these effects have been estimated in previous studies ${ }^{(5)}$ to account for less than $10 \%$ of the mean freestream velocity. The difficulty in making near wall measurements with a larger and hence more-intrusive cross- or triple-wire sensor also makes the use of a single wire boundary layer probe a more attractive option.

\subsection{RESULTS AND DISCUSSION}

The unmodified boundary-layer mean velocity profile at $x / d_{\mathrm{o}}=10$, with freestream velocity $U_{\infty}=28 \mathrm{~ms}^{-1}$, is illustrated in Fig. 4. This was measured at 11 spanwise positions; three are illustrated here. The boundary layer was turbulent upstream of the actuator position, downstream of the transition strip. The boundary thickness at

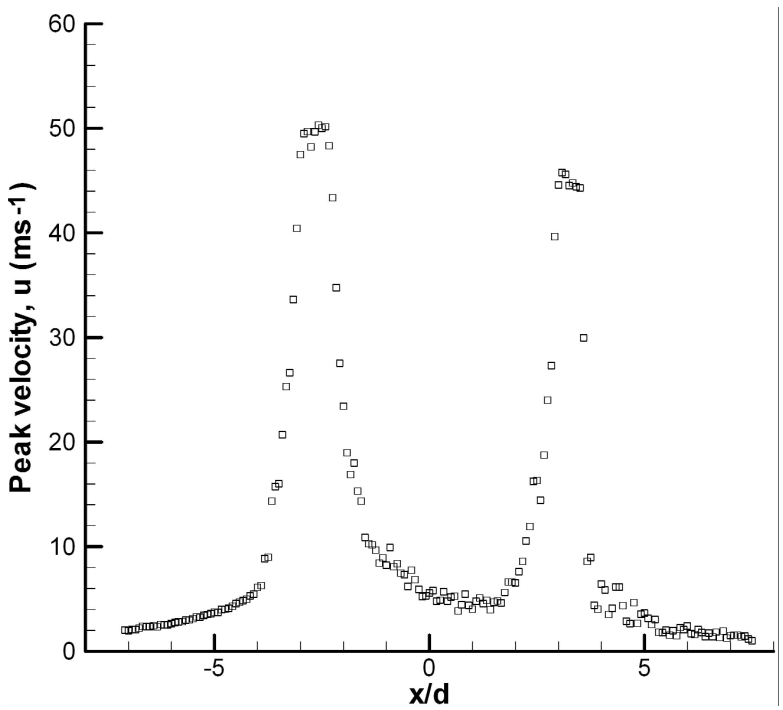

Figure 5. 2-SJA configuration array peak velocity performance, measured at $y / d_{0}=1$.

$x / d_{\mathrm{o}}=10$, expressed non-dimensionally, was $4 \cdot 25 d_{\mathrm{o}}$.

Prior to the boundary-layer experiment, the performance of the actuators was measured in quiescent conditions, using CTA. The peak velocity performance of the two actuators, set up for a nominal velocity ratio of $R=2$, is illustrated in Fig. 5. As stated above, the performance was matched using variation of the input signal amplitude. The peak velocity was obtained using hotwire anemometry at a distance equal to the orifice diameter above the surface. Where dimensions are stated subsequently, these are nondimensionalised with respect to the orifice diameter.

Although this work is primarily concerned with the downstream effects of the SJAs, it is convenient to consider the near-orifice region in order to analyse the flow-visualisation data. This analysis is based on the previous work reported by $\operatorname{Crook}^{(7)}$. A typical oilflow visualisation result for a single actuator is illustrated in Fig. 6, in order to demonstrate the salient points. Upstream of the orifice, strong suction is evidenced by the radial entrainment patterns. At the downstream edge of the orifice, this pattern is altered due to the reversal of the suction direction relative to the boundary-layer velocity; two distinct scrubbed regions therefore remain, as evidence of periodic flow reversal. In between these two regions, a central bright region is visible, lacking distinct structures, and corresponding to the periodic movement of distinct vortex rings. The reformation of these discrete flow features into a pair of streamwise vortices is evidenced by the resulting downstream patterns.

\subsection{ORIFICE YAW}

A typical, non-phase shifted visualisation of Configuration 2 is illustrated in Fig. 7. This baseline case of in-phase input signals demonstrates that the path of the upstream jet appears to have been deflected towards the downstream orifice ${ }^{(1)}$. Further downstream, at distances normalised by orifice diameter of $\mathrm{O}^{(10)}$, the left-hand-most structure is deflected in the opposite direction ${ }^{(2)}$, and appears to proceed on a straight path angled away from the right-hand-most structures, which are aligned almost parallel with the freestream direction $^{(3)}$. Both of these structures show significant spreading downstream. The central structures are clearly dominant ${ }^{(4)}$; from this evidence the conclusion that destructive interaction, as has previously been suggested ${ }^{(6)}$, and which would manifest itself in the repression of streamwise flow structure formation near to the centreline of the configuration, cannot be confirmed. 


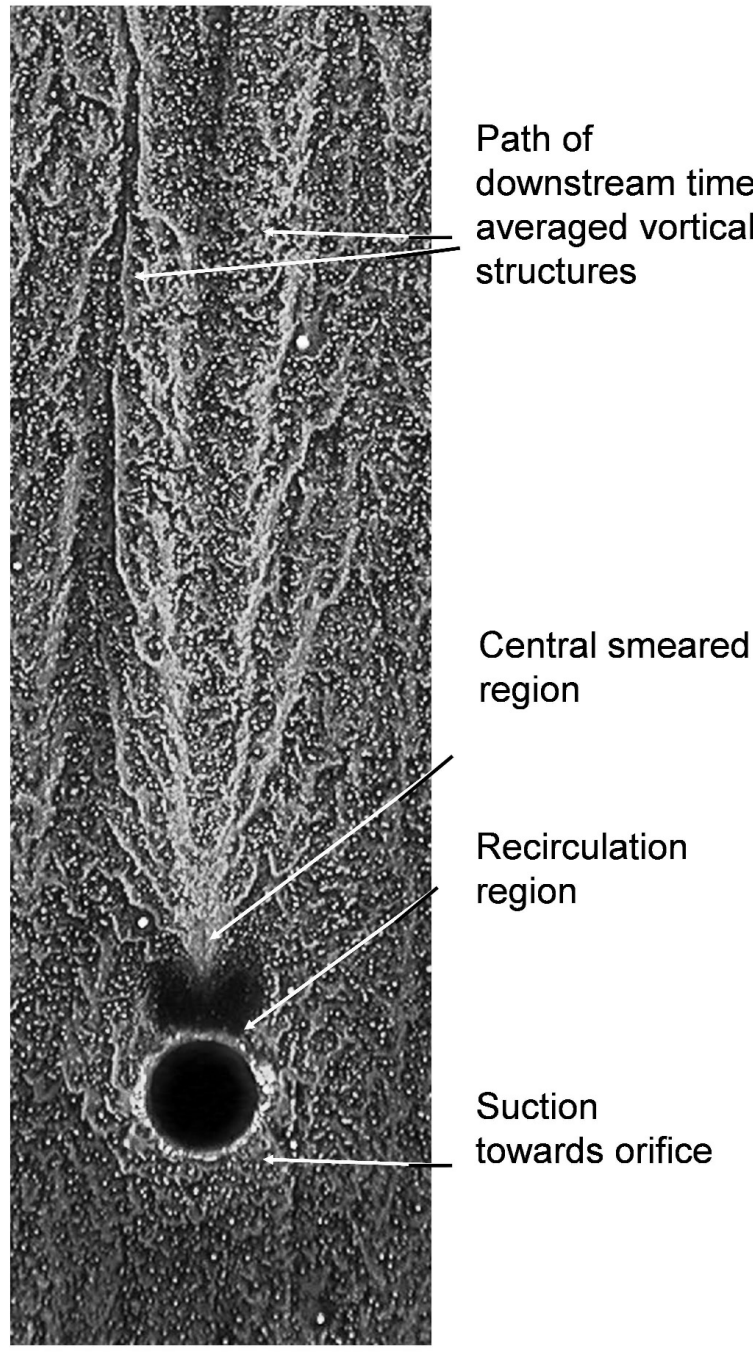

Figure 6. Typical oil-flow visualisation image of an SJA in a zero-pressure gradient boundary layer. Flow from bottom to top. $U=28 \mathrm{~ms}^{-1}, f=, 1,950 \mathrm{~Hz}, R=1.59$.

Figure 8 illustrates time-averaged streamwise velocity contours downstream of the two configurations. Velocities are given in nondimensional form, with respect to the freestream. Considering first the streamwise orientation, Configuration 3, the presence of the synthetic jet is obvious. Centred on the actuator axis, $z / d_{\mathrm{o}}=0$, low speed fluid evidently from the near wall region has been swept upwards. At the same time, at virtually symmetrical positions offcentre, at approximately $z / d_{\mathrm{o}}= \pm 2$, the higher-velocity fluid from the mid-region of the boundary layer has been swept downwards. The virtually symmetrical pattern is indicative of the presence of a counter-rotating vortex pair (CRVP). This pattern is repeated with increasing downstream distance, with the vertical penetration of the vortex also increasing. In the $15 d_{\mathrm{o}}$ downstream distance considered here, the increase in maximum height of the velocity deficit region, i.e. that in which redistribution of streamwise momentum has been undertaken, increases by at least one non-dimensional unit. The contours close to the wall on the centreline exhibit less up-sweep as downstream distance is increased, possibly indicative of the vortex moving away from the wall. Along the measurement space

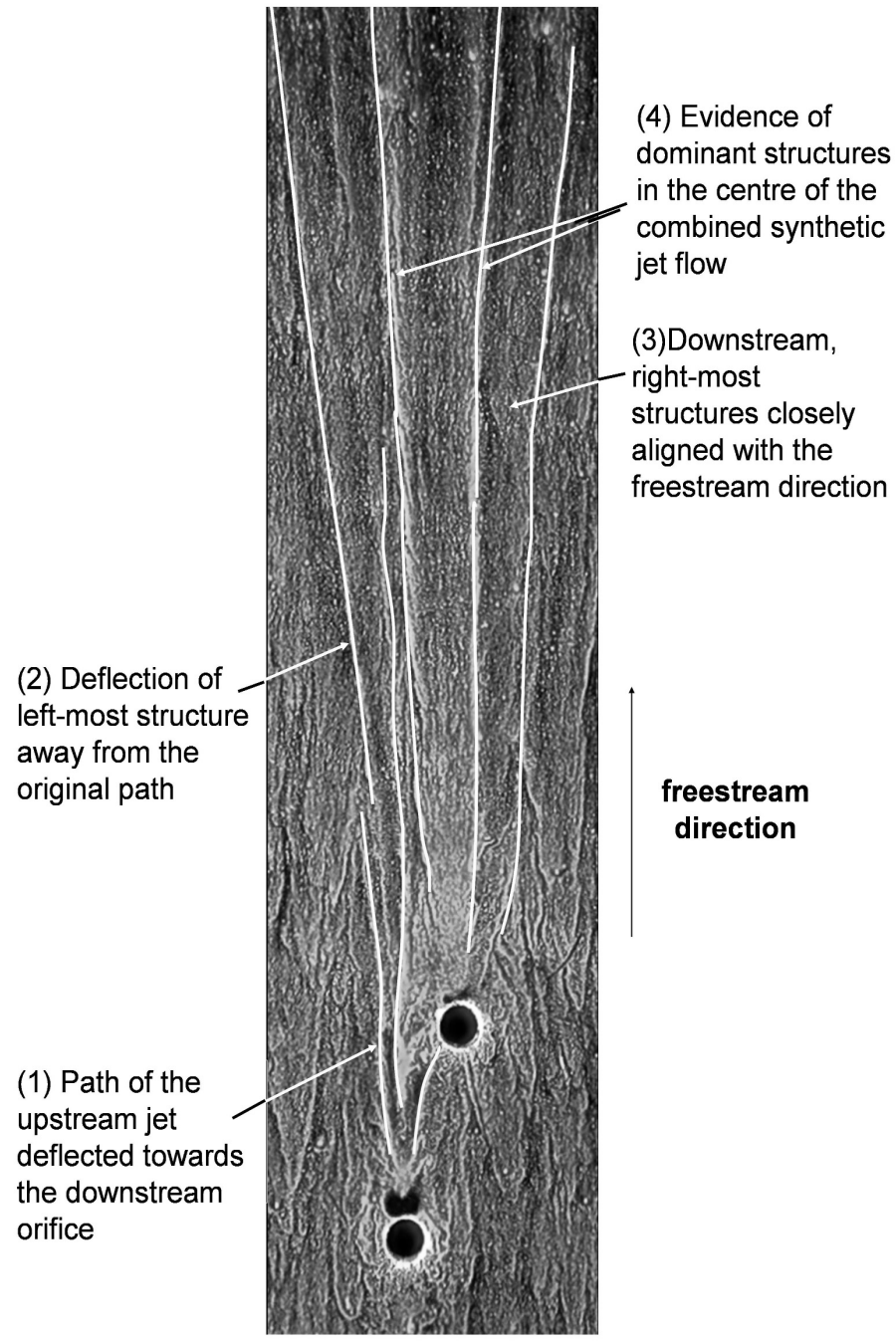

Figure 7. Oil-flow visualisation of two SJAs with axis at $75^{\circ}$ to spanwise datum. $U=28 \mathrm{~ms}^{-1}, f=1,950 \mathrm{~Hz}, R=1.59$ and $\phi=0^{\circ}$.

considered, there is little change in the spanwise extent of the synthetic jet's influence within the boundary layer.

As might be expected from reference to the oil-flow visualisation data, the corresponding contours for Configuration 2 are notable for their asymmetry. The lack of two independent patterns similar to the Configuration 3 contours confirms that an interaction between the two synthetic jets has taken place. The downstream orifice position is to the left of the centreline, with a broadly similar pattern of upswept low-momentum flow as seen on the centreline of configuration 3 at the same downstream distance. At the spanwise position of the upstream actuator, however, the familiar pattern within the boundary layer is not exhibited. At the edge of the boundary layer, upsweep of fluid can be seen to have occurred. The lack of downswept fluid toward the right (positive $y / d_{\mathrm{o}}$ ) of the contour plots suggests that the resultant flow is not a single vortex, although it may be observed that at the extreme right of the measurement space some small redistribution downwards can be seen, possibly relating to the extended distance from the centreline of the RHS structure visible noted in Fig. 7. Certainly, the influence of the configuration 

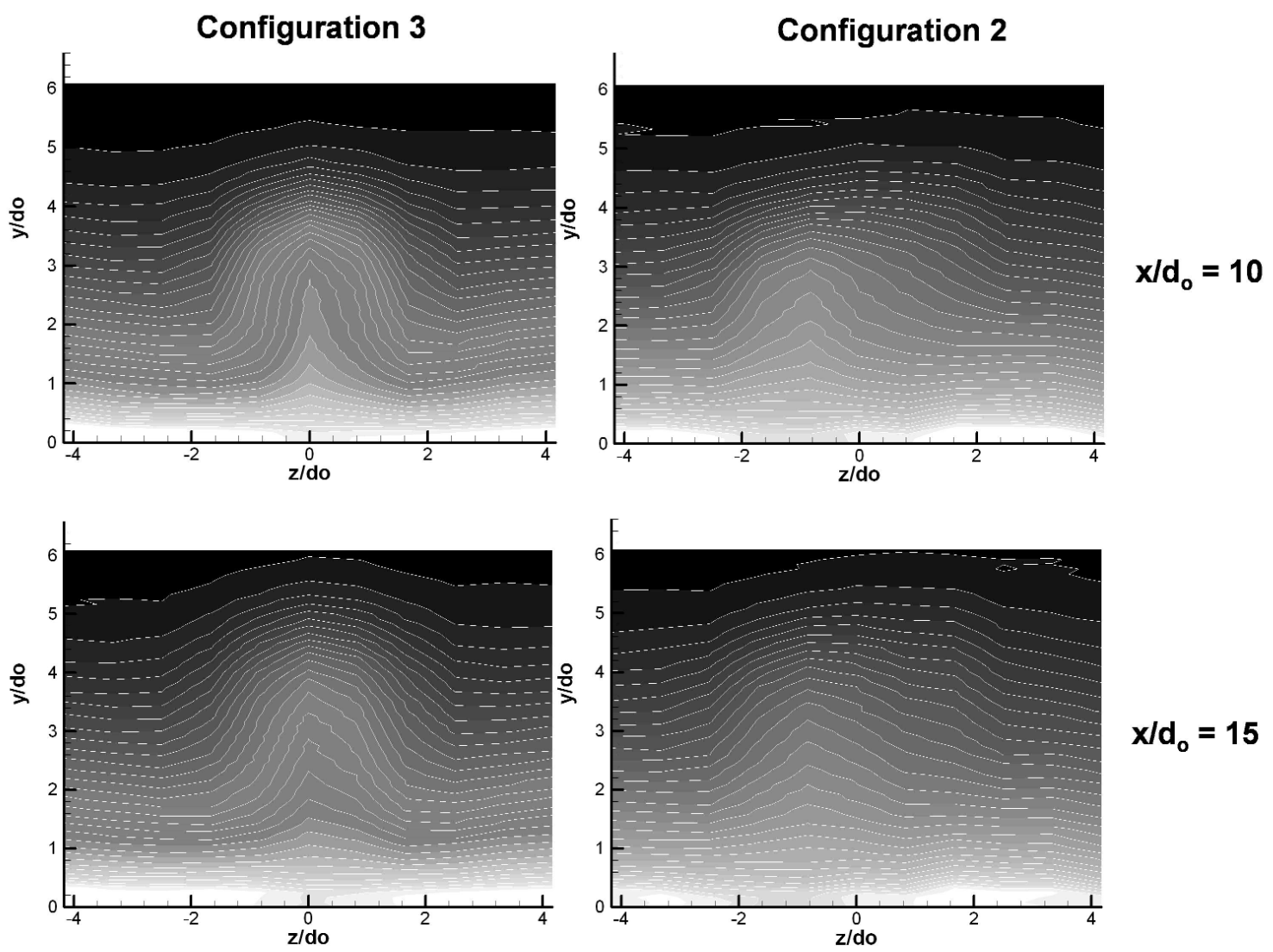

$x / d_{0}=15$
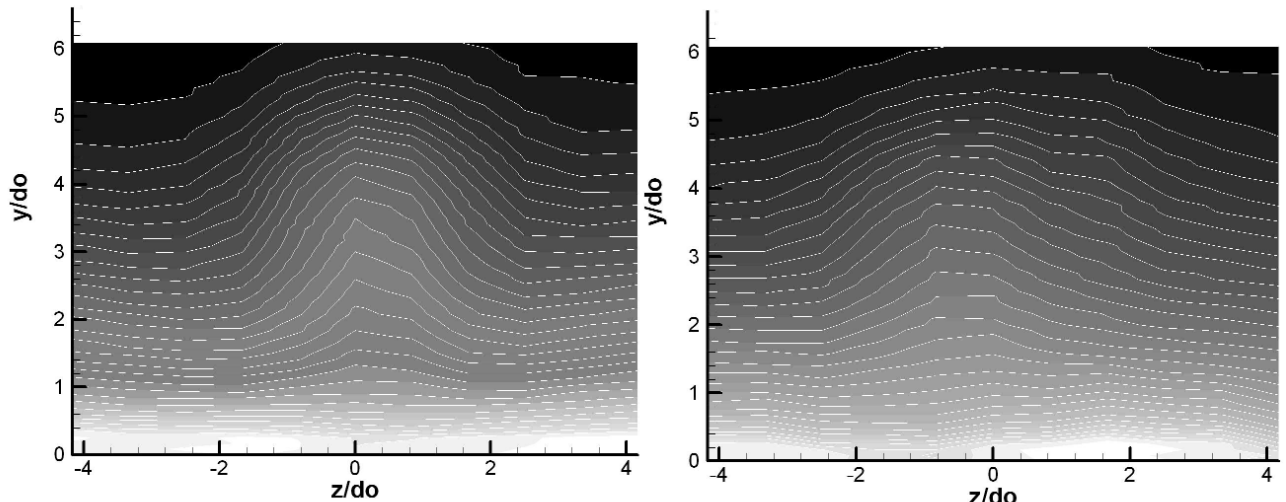

$x / d_{0}=20$
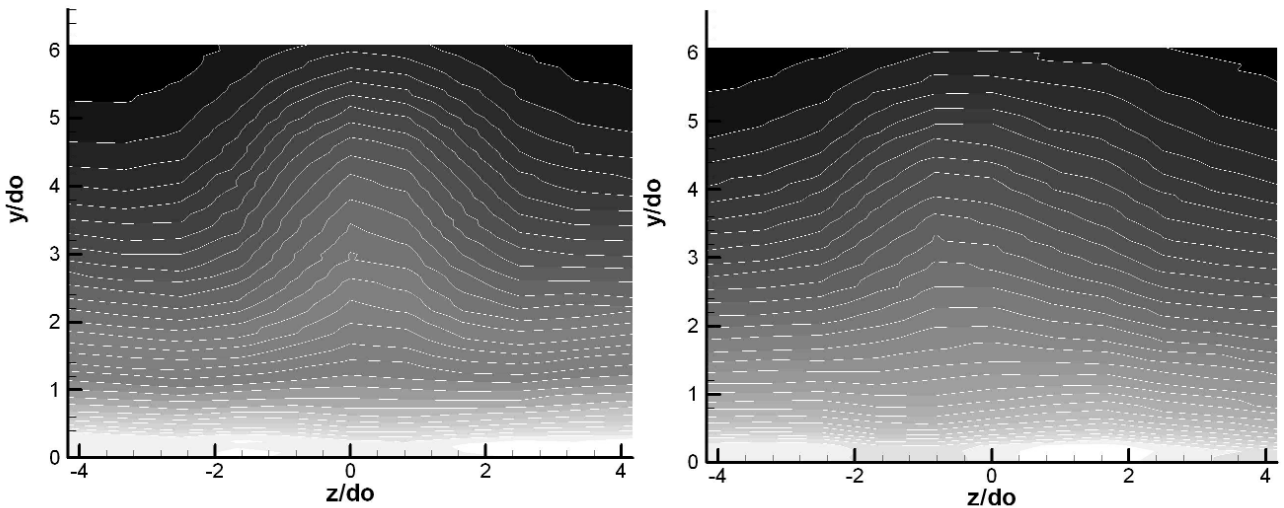

$x / d_{0}=25$

wU: 0.5830 .5970 .6110 .6250 .6390 .6530 .6670 .6810 .6550 .7090 .7230 .7370 .7510 .7650 .7790 .7930 .8070 .8210 .8350 .8500 .8640 .8780 .8920 .9060 .9200 .9340 .9480 .9620 .9760 .990

Figure 8. Time-averaged streamwise velocity contours, downstream of (left)

Configuration 3 and (right) Configuration 2. $U=28 \mathrm{~ms}^{-1}, f=1,600 \mathrm{~Hz}, R=2$ and $\phi=0^{\circ}$. 
extends along a much larger spanwise distance than that of configuration 3. In each case vertical penetration, although slightly reduced, is of similar order.

From this data therefore, it is possible to conclude that the result of the synthetic jet-boundary layer interaction in the case of Configuration 3 is a pair of counter-rotating vortices, extending beyond $25 d_{\mathrm{o}}$ downstream. With increasing downstream distance, the influence of the interaction extends upwards out of the boundary layer and exhibits spreading.

In the case of Configuration 2, the interaction extends over a wider spanwise range, with the focus slewed towards the downstream actuator. Although the oil-flow visualisation shows streak-lines on the upstream actuator side, the hotwire analysis confirms only that, by virtue of asymmetry, structures from this actuator influence the development of the downstream synthetic jet.

\subsection{INPUT SIGNAL RELATIVE PHASE}

Surface flow visualisation of the effect of four relative phase changes: $0^{\circ}$ (in-phase), $90^{\circ}, 180^{\circ}$ and $270^{\circ}$, is illustrated in

Figure 9. considering configuration 2 initially, the deflection of the left-hand structure described above towards the downstream orifice can be seen to be lessened in the $180^{\circ}$ and $270^{\circ}$ cases when compared to the in-phase and $90^{\circ}$ cases. However, more obvious changes are visible in the case of Configuration 3. An incremental increase in the spreading rate of the outermost structures is visible between the in-phase and $180^{\circ}$ cases. The latter case shows separation into four distinct paths, also visible to a lesser extent in the $90^{\circ}$ case. However, the $270^{\circ}$ case shows a narrower path, spreading significantly downstream.

Further investigation of the downstream flow structure was undertaken using CTA. This has enabled analysis of the flow away from the surface and illustrated the effect of input signal phase on the flow structure.

Figure 10 shows time-averaged velocity contours measured at $x / d_{\mathrm{o}}=10$ and $x / d_{\mathrm{o}}=25$. The baseline $\phi=0^{\circ}$ case is again illustrated for ease of comparison. As noted above, the presence of the jet in each case is evidenced by a region of fluid featuring a velocity defect, almost centrally located within the measurement space. It is notable that at this streamwise location, any effect of phase difference has not manifested itself in a significant change in the width of the structure, which remains a CRVP. It should be noted that the spanwise resolution of the measurement is $1 \mathrm{~mm}$, which is comparable with the orifice diameter. Significant spanwise effect is limited to 3-4 diameters in all cases.

Compared with the baseline case, the $\phi=90^{\circ}$ features more abrupt changes in contour. The upsweep of fluid extends to greater vertical distances at both streamwise locations. In both of the $\phi=$ $180^{\circ}$ and $\phi=270^{\circ}$ contour plots, and at both streamwise locations, the penetration of the structure is significantly reduced. At $x / d_{\mathrm{o}}=10$, both of these cases are comparable with the baseline, although at the extreme downstream location the $\phi=270^{\circ}$ case exhibits the lowest penetration of those considered.

Although the streamwise velocity contours show somewhat limited differences, the fact that relative input signal phase does have an effect on the resultant flow is provided by Power Spectral Density analysis of the hotwire velocity time-histories, as illustrated in Fig. 11. As an example, data for $x / d_{\mathrm{o}}=10$ and $y / d_{\mathrm{o}}=1$ are given. Similar patterns are visible for the $\phi=0^{\circ}, 90^{\circ}$ and $180^{\circ}$, with a single large peak at the $1,600 \mathrm{~Hz}$ actuation frequency, indicative of the passage of some discrete periodic structures from the actuators. The magnitude of this peak is largest in the $\phi=90^{\circ}$ case. The result for the $270^{\circ}$ case is very different, in that the most significant peak is that occurring at twice the value of the actuation frequency, with a magnitude of less than half of that for the major peaks in other cases; a smaller peak is found at $1,600 \mathrm{~Hz}$.
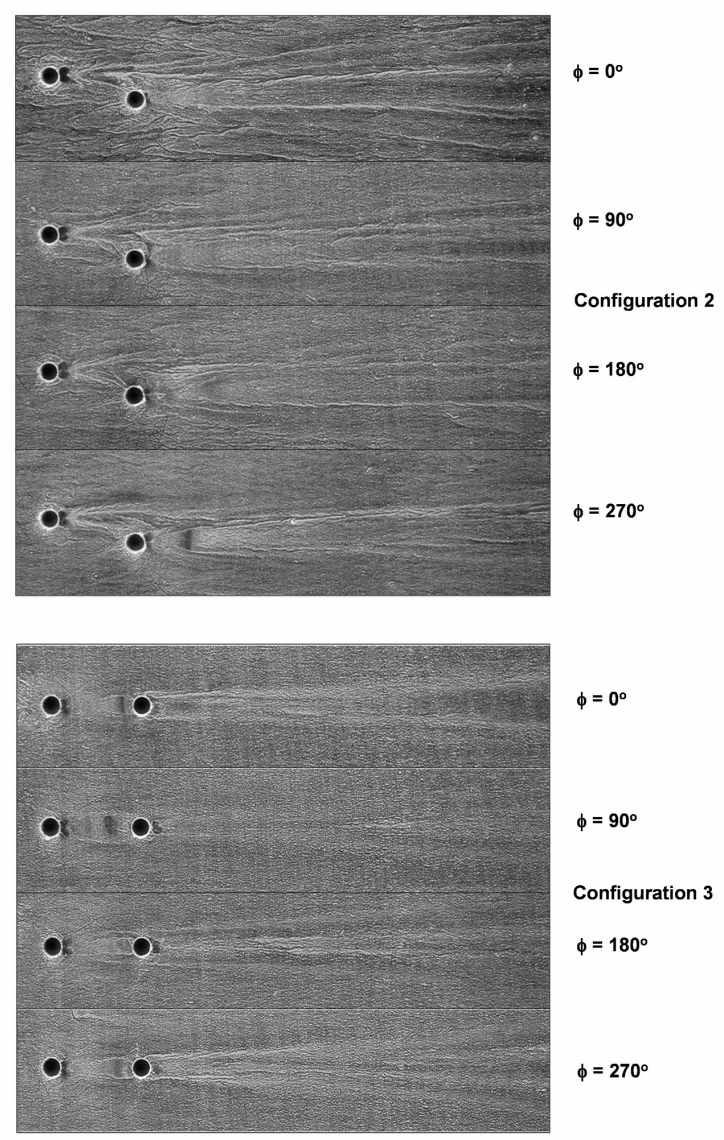

Figure 9. Flow visualisation of two SJAs with (upper) Configuration 2 and (lower) Configuration $3 . U=28 \mathrm{~ms}^{-1}, \mathrm{f}=1,950 \mathrm{~Hz}, R=1 \cdot 54$. Phase shift of upstream relative to downstream input signal in each case.

A proposed explanation for the above observations is that the combination of orifice spacing and convective velocity of the vortex rings from the two actuators results in the initial creation of two separate counter-rotating vortex pairs, obviously inducing velocities on each other. The development of a combined streamwise structure is shown to have occurred by $x / d_{\mathrm{o}}=10$ in Fig. 8 . The variation in development would be influenced by the relative positions of pairs of individual vortex rings, one from each orifice, at a given time. For flow control applications, this is of particular significance as the stability of the resulting structure will be affected. In these applications, the ability to keep the structures within the boundary layer and hence causing mixing of low- and high-momentum fluid is paramount, and the behaviour of the CRVP must be understood.

\subsection{CONCLUSIONS}

A series of experiments have been undertaken in order to detect and visualise the behaviour of fluidic structures produced by two interacting small-scale Synthetic Jet Actuators. Oil-flow visualisation and CTA techniques were used to investigated the flow downstream of two SJA configurations in a zero-pressure gradient boundary layer on a flat plate, at two yaw angles. This has demonstrated:

- The mode of interaction between two synthetic jets and a turbulent boundary layer can be controlled by configuration yaw angle. This 

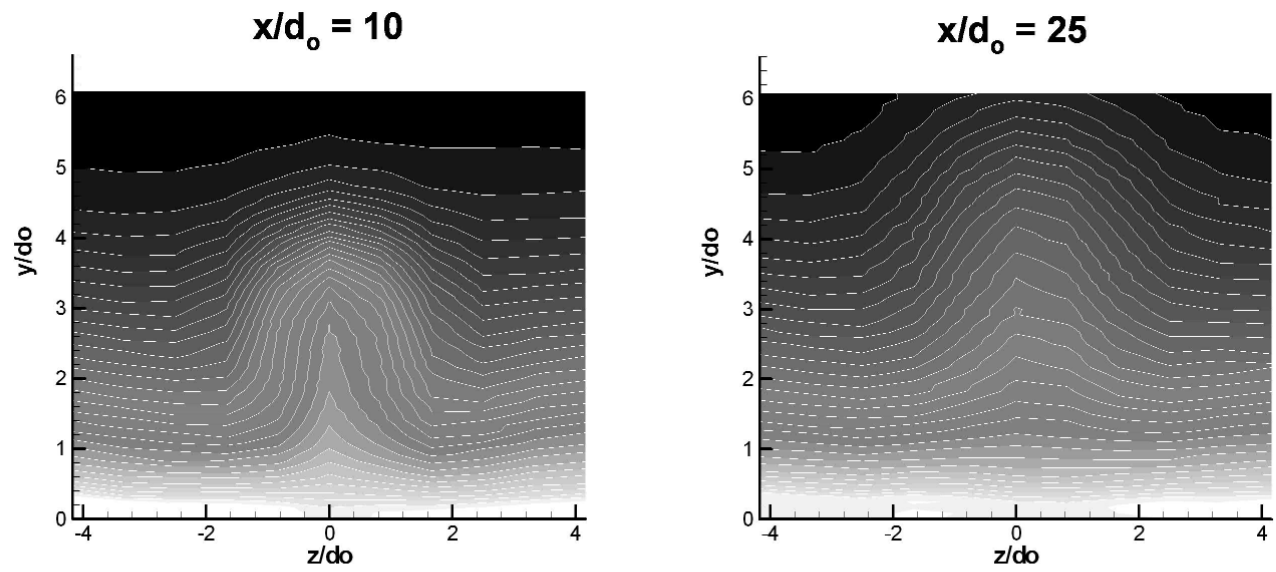

$$
\phi=0^{\circ}
$$
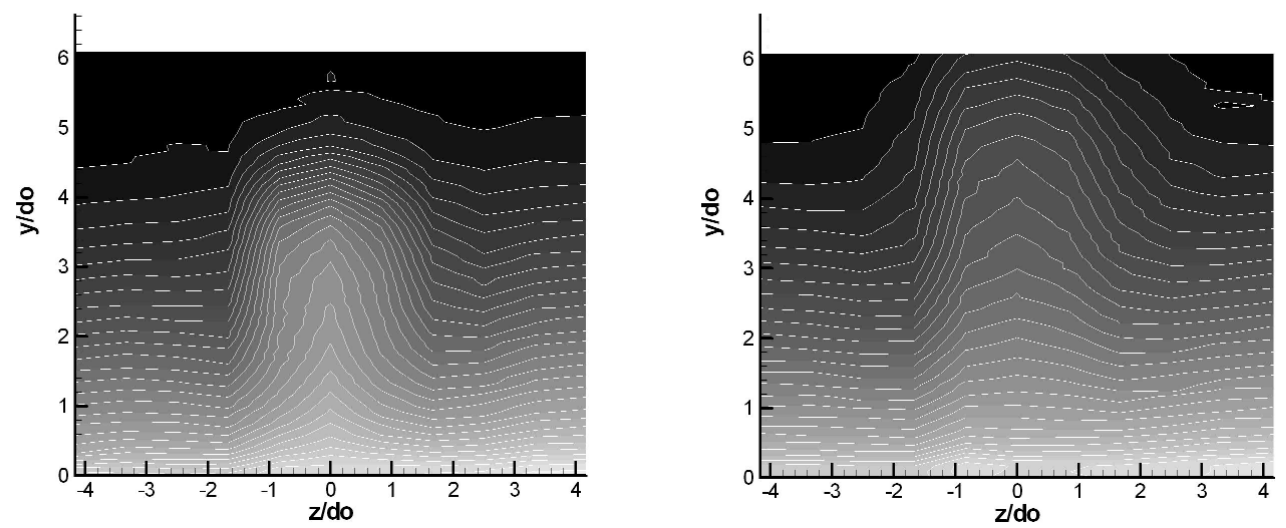

$$
\phi=90^{\circ}
$$
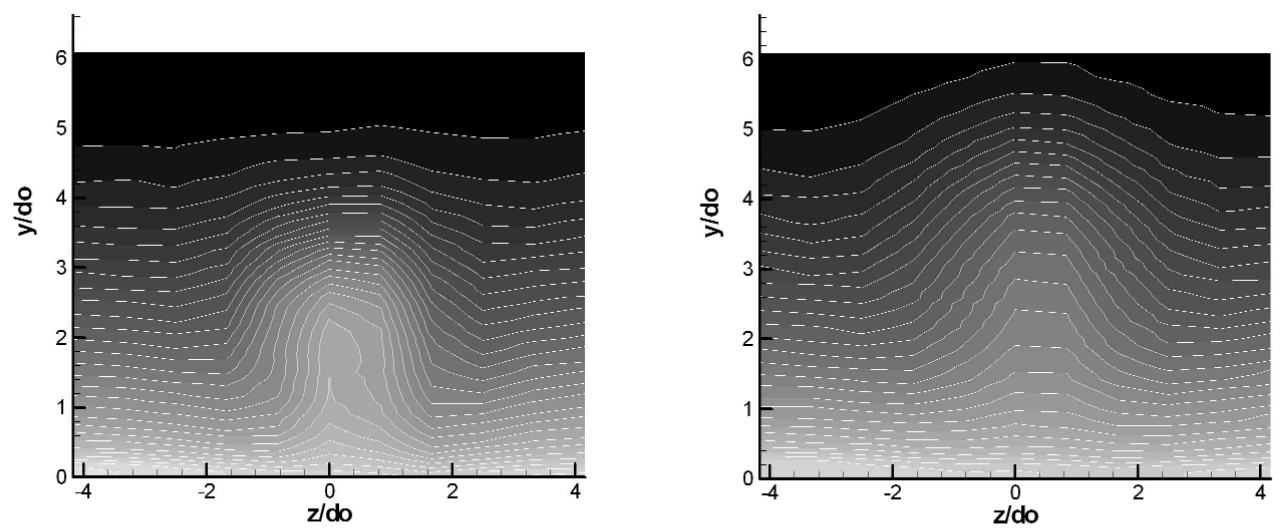

$$
\phi=180^{\circ}
$$
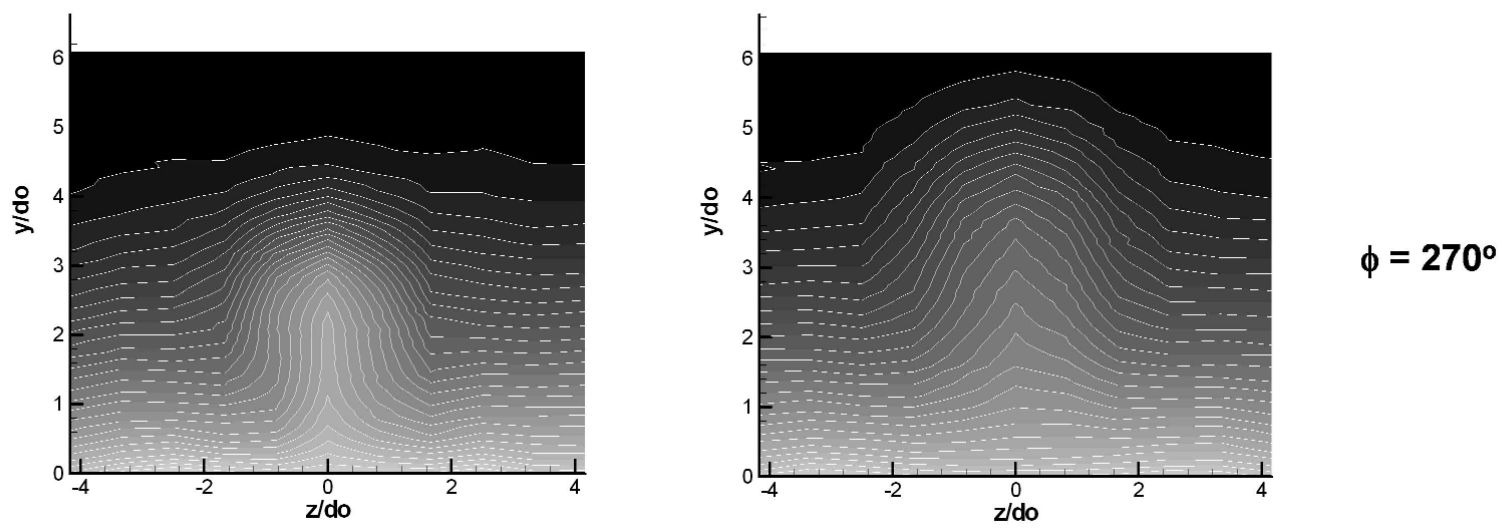

Figure 10. Time-averaged streamwise velocity contours at (left) $x / d_{0}=10$ and (right) $x / d_{\mathrm{o}}=25$ for Configuration 3. $\mathrm{U}=28 \mathrm{~ms}^{-1}, f=1,600 \mathrm{~Hz}$ and $R=2$. 

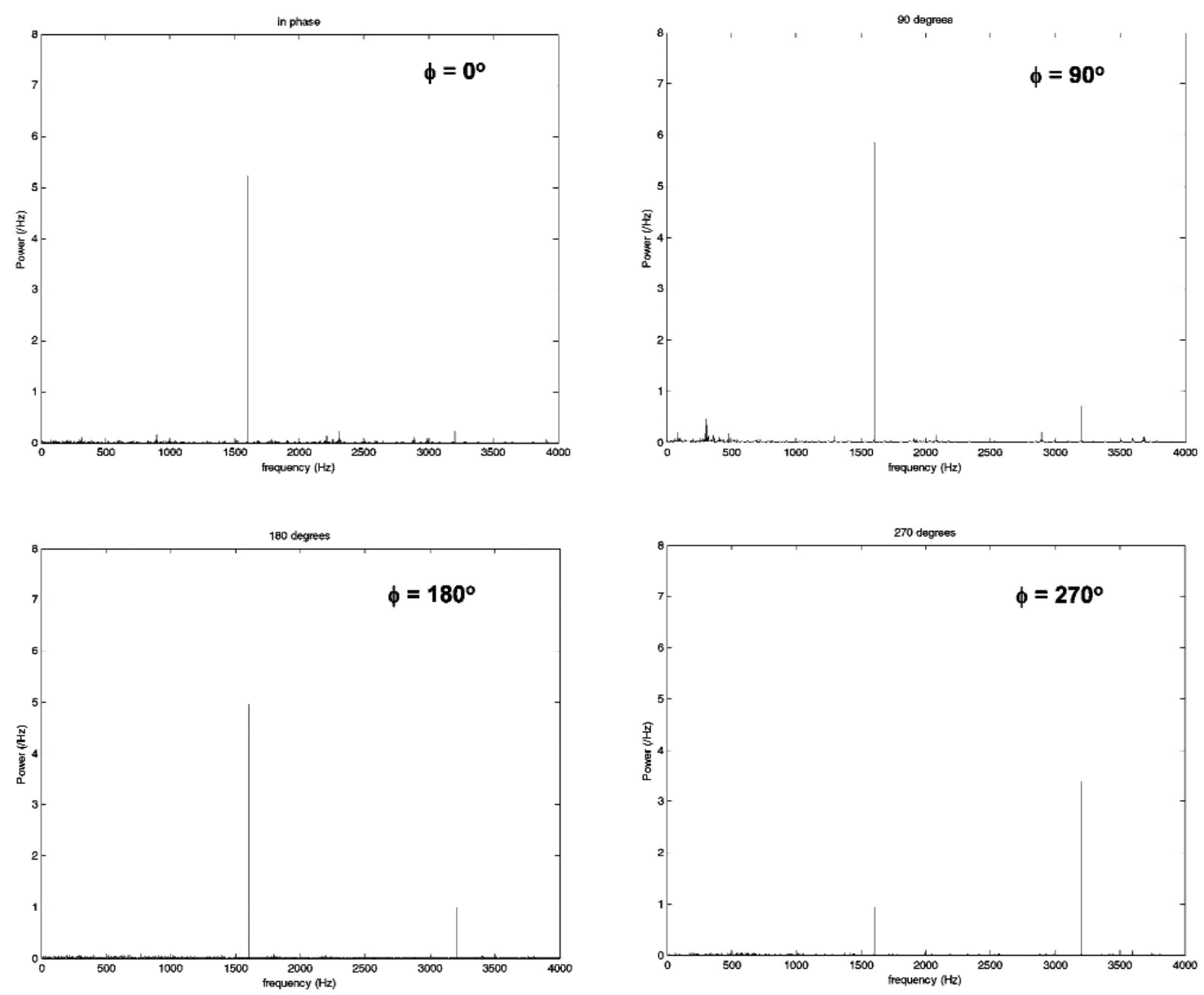

Figure 11. PSD plots of hotwire anemometer output signal, measured at $x / d_{0}=10 d, y / d_{0}=1$ for Configuration 3 . Clockwise from top left: $\phi=0^{\circ}, 90^{\circ}, 270^{\circ}$ and $180^{\circ} . U=28 \mathrm{~ms}^{-1}, R=2$ and $f=1,600 \mathrm{hz}$.

was illustrated using oil-flow visualisation and streamwise velocity contour mapping at four downstream locations

- The variation in downstream structures between four equi-spaced input signal phase differences was visible in the surface oil-flow visualisation of the two SJA configurations considered.

- Velocity profiles were presented, illustrating that in all cases, the effects of the synthetic jet flow on the boundary layer, measured at $x / d_{\mathrm{o}}=10$, were evident over a similar spanwise range. This was of the order $3 d_{\mathrm{o}}-4 d_{\mathrm{o}}$, with the evident CRVP both spreading and moving away from the surface. This effect was maximised in the $\phi=90^{\circ}$ case; the $\phi=270^{\circ}$ appeared to remain closer to the surface and showed greater interaction with the immediate nearwall flow.

- PSD analysis of the downstream velocity time-histories showed distinct differences in the $270^{\circ}$ case, with a major peak at a value of twice the actuation frequency, compared with the three other cases considered in which the major peak occurred at the actuation frequency.

- The difference in penetration of the synthetic jet structure is likely to be influenced by the relative velocities induced by adjacent pairs of vortex rings; the relative signal input phase controls this relative position.
For the flow-control applications considered for synthetic jets, the stability of the resulting downstream structures is of vital importance in allowing effective mixing of low and high momentum fluid.

Future investigation will establish the time-variant flow structures, by using phase-locked CTA measurement.

\section{ACKNOWLEDGEMENTS}

The authors would like to recognise the contributions of Goldstein Laboratory technical staff in manufacturing the rig components and Bilkay Gulacti for experimental assistance. The project is supported by Airbus and EPSRC.

\section{REFERENCES}

1. Amitay, M., Smith, D.R., Kibens, V., Parekh, D.E. and Glezer, A. Aerodynamic flow control over an unconventional airfoil using synthetic jet actuators; AIAA J, March 2001, 39, (3), pp 361-370.

2. PACK, L.G., Schaeffler, N.W., Yao, C.S. and Seifert, A. Active control of separation from the slat shoulder of a supercritical aerofoil, AIAA Paper, June 2002, pp 2002-3156. 
3. WATSON, M., JAWORSKI, A. J. and Wood, N.J. A study of synthetic jets from rectangular and dual-circular orifices, Aeronaut J, 107, (1073) July 2003, pp 427-434.

4. SMith, D.R. Interaction of a synthetic jet with a crossflow boundary layer, AIAA J, November 2002, 40, (11), pp 2277-2288.

5. BRIDGES, A and SMITH, D.R. Influence of orificce orientation on a synthetic jet-boundary layer interaction, AIAA J, December 2003, 41, (12), pp 2394-2402.

6. WatSON, M., JAWORSKI, A.J. and WoOD, N.J. Contribution to the understanding of flow interactions between multiple synthetic jets, AIAA J, 2003, 41, (4), pp 747-749.

7. Сroок, A. PhD Thesis, 2002, University of Manchester, UK.

8. Morgan, H.L., Experimental test results of the energy efficient transport (EET) flap-edge vortex model in the langley low-turbulence pressure tunnel, NASA TM-2002-211928, 2002.

9. Lockerby, D.A. and CARPENTER, P.W. Modeling and design of microjet actuators, AIAA J, February 2004, 42, (2), pp 220-227.

10. Amitay, M. and Glezer, A. Role of actuation frequency in controlled flow reattachment over a stalled aerofoil, AIAA J, 40, (2) February 2002, pp 209-216. 